Dossiê: América Latina como lugar de enunciação

\title{
ENCARTE DIGITAL
}

\section{Entrevista com César Germaná Cavero}

\section{Interview with César Germaná Cavero}

\author{
Adelia Miglievich-Ribeiro*
}

O professor César Germaná Cavero, sociólogo peruano, formou-se na Universidad Nacional Mayor de San Marcos, en Lima, tendo seu mestrado na Facultad Latinoamericana de Ciências Sociais do Chile e doutorado na Universidade de Stdhande Francia. É catedrático em San Marcos e, hoje, conta mais de 30 anos como pesquisador e formador de gerações de pesquisadores.

Referência certa para os estudiosos do "socialismo indoamericano de Mariátegui", título também de seu livro. Dedicou-se ainda às investigações acerca das transformações no mundo do trabalho e a pensar os desafios contemporâneos da produção de conhecimento no continente latino-americano, atentando para um modelo de universidade que contemple as demandas das interculturalidades e das descolonialidades.

Tive a honra de entrevistá-lo em Lima, cumprindo uma promessa antiga quando coordenamos juntos um grupo de trabalho no congresso da Alas (Associação Latino-Americana de Sociologia), realizado em Recife no ano de 2011. Na ocasião, Germaná, atento a meu vivo interesse pelo exílio de Darcy Ribeiro e seus "fazimentos", chamou-me a atenção para sua presença no Peru quando do Governo Velasco Alvarado, entre fins de 1960 e início dos anos 1970. O intelectual brasileiro teria atuado para reforma universitária peruana. Darcy Ribeiro reconhecera num governo militar nacionalista, num paradoxo apenas aparente, uma estratégia de enfrentamento do capital internacional e

\footnotetext{
* Doutora em Sociologia pela Universidade Federal do Rio de Janeiro (UFRJ, RJ, Brasil), professora do departamento de Ciências Sociais, do PPG em Ciências Sociais e do PPG em Letras da Universidade Federal do Espírito Santo em Vitória, ES, é bolsista produtividade do CNPq. Fez pós-doutorado (PDS- Faperj) no PPG em Educação da Universidade do Estado do Rio de Janeiro (Uerj)<miglievich@gmail.com>. A entrevista foi realizada no dia 24 de janeiro de 2014 e contou com a participação especial da professora Sara Beatriz Guardia. Transcrição: Valentina Zuliani.
} 
de seus aliados locais, ao mesmo tempo em que via avanços concretos em prol dos setores populares e dos trabalhadores rurais - a maioria - com a criação da "propriedade social". Sob o lema de "devolver o Peru ao povo peruano", Velasco convenceu Darcy Ribeiro e outros intelectuais de esquerda expurgados de seu próprio país pelas forças reacionárias a somarem-se ao novo empenho naqueles conflituosos anos.

Tivemos uma agradável conversa. Aprendi sobre o Peru e sobre o velasquismo em particular. Trocamos ideias sobre a universidade e os novos desafios, sobretudo quando o ensino superior se transforma em "negócio". Na contracorrente, temos a hercúlea tarefa de descolonizar a universidade. Volta e meia, recordávamos Darcy Ribeiro e sua "universidade necessária”.

Germaná, devo dizer, é um dos intelectuais mais brilhantes, gentis e generosos que pude conhecer, o que me dá a certeza de que o intercâmbio universitário em nossa vasta América Latina é promissor e valioso.

Registro aqui meu agradecimento, também, à delicadeza da oferta de sua casa para que o encontro acima pudesse ocorrer por parte da professora e escritora peruana Sara Beatriz Guardia. 


\section{Conversando com César Germaná sobre o Peru dos anos 1960-70, a presença de Darcy Ribeiro em Lima, os destinos da universidade: porque a interlocução é necessária}

- Adelia Miglievich-Ribeiro: César, estoy estudiando a Darcy Ribeiro como usted ya sabe, y particularmente la presencia de Darcy Ribeiro en Perú, cuando él estaba en su exilio. Cerca de 1970 Darcy Ribeiro fue invitado para trabajar acá. ¿En esta época usted conoció a Darcy Ribeiro?

- César Germaná: No, no lo conocí. Tenía referencias de la existencia de Darcy, de los trabajos que realizaba y de su vinculación con algunos intelectuales que apoyaron el régimen militar del general Velasco.

- Adelia Miglievich-Ribeiro: ¿Dónde estaba usted en este momento en $1970 ?$

- César Germaná: En 1970 comencé a estudiar en la Universidad de San Marcos y hubo un momento en donde podría haberme incorporado al Sinamos (Sistema Nacional de Apoyo a Movilización Social). Carlos Franco que ya falleció era bastante amigo y más que todo el hermano de Carlos Delgado, director do Sinamos, donde estaba trabajando Darcy Ribeiro. Carlos (Franco) me invitó a participar en el Sinamos pero yo estaba en otro proyecto, en el de "Sociedad y Política" con Aníbal Quijano, que éramos más bien críticos del régimen militar.

- Adelia Miglievich-Ribeiro: ¿Usted estaba entre los críticos del gobierno Velasco?

- César Germaná: Del gobierno militar, sí. Y publicábamos una revista de la que salieron 13 números, que se llamaba "Sociedad y política", que en ese momento tuvo un impacto muy grande porque en la izquierda no había una idea clara de lo que significaba el régimen de Velasco.

- Adelia Miglievich-Ribeiro: Después de tantos años ¿Hay hoy una mayor claridad sobre el gobierno de Velasco? ¿Sobre lo que representó para la sociedad peruana?

- César Germaná: Yo creo que sí. En la actualidad se puede tener una percepción más justa de lo que fue el régimen militar de Velasco, yo creo que corresponde a un ciclo que se inició hacia 1930, lo que podría ser el equivalente a la revolución francesa. Hay una revolución democráticoburguesa pero no dirigida por la burguesía sino por las capas medias de la población. El APRA de los años 30, creo que encarnó claramente ese 
proyecto, que era un proyecto anti-imperialista y anti-oligárquico, quería la independencia nacional y la democratización de la sociedad peruana. El APRA después de 1940 abandona ese proyecto, pero va a ser retomado por las nuevas capas medias, profesionales y ciertos sectores militares; y se va a completar esa nueva versión con el régimen militar. O sea, el régimen militar de Velasco puede ser pensado como el punto culminante de ese proceso que se inició en los años 30 . Y por eso el APRA, de alguna forma reivindicaba el régimen militar, Haya de Latorre y varios de esa época decían que era eso lo que ellos habían planteado, aunque lo que faltaba era la participación del pueblo. Y el asesor principal de Velasco fue Carlos Delgado que había sido secretario privado de Haya de Latorre. Entonces ese proyecto anti-imperialista y anti-oligárquico lo plasmó el velasquismo, con el velasquismo se cierra una época, la época de la sociedad oligárquica, y se abre otra que es la época en que vivimos.

En Perú con retraso se produce esa transformación que ya se había dado en Argentina o Brasil, desde el año 30 en Brasil ya había un movimiento anti oligárquico con Vargas, que es lo que ocurre aquí con Velasco.

- Adelia Miglievich-Ribeiro: Darcy Ribeiro fue invitado por Velasco porque ya era considerado un reformador de la universidad. ¿Cuál es su opinión sobre la personalidad de Darcy Ribeiro?

César Germaná: Bueno a mí me parece que es uno de los intelectuales más importantes de América Latina, y creo que el esfuerzo fundamental fue el de desarrollar un pensamiento autónomo, y en eso coincido contigo en la idea de que allí se encuentran raíces de lo descolonial o postcolonial, aunque en ese momento él no plantea en ninguno de sus escritos la idea de la postocolonialidad ni la de la descolonialidad. Pero lo que sí hay es una búsqueda de una identidad latinoamericana, y creo que dentro de esta perspectiva se acercó o aceptó participar del proyecto de la reforma de educación del régimen militar. Precisamente este libro "La universidad peruana" fue un producto de un periodo de lo que en ese momento se llamaba el consejo superior de la universidad peruana (Conup).

Entonces el esfuerzo fue el de llevar adelante un proyecto de reforma de la universidad, yo creo como para muchos, el régimen militar de Velasco significó un proceso, como ellos mismos se autodenominaban, revolucionario, es decir, ahí hubo un cambio profundo de la sociedad peruana; la reforma agraria y la nacionalización de las empresas extranjeras, dio un vuelco en la prensa. Pero, por eso creo que Darcy Ribeiro aceptó venir al Perú en la medida que veía un proceso de cambio, de transformación, por 
lo tanto era necesario, como él señala en varios textos sobre la Universidad, era necesario un proyecto educativo en función de las transformaciones de la sociedad. Es decir, veía que las universidades conservadoras eran su preocupación...

- Adelia Miglievich-Ribeiro: ¿La innovación de Córdova tenía repercusión en la universidad peruana?

- César Germaná: Sí, el proceso de Córdova fue bastante complejo aquí en el Perú. En 1920 se da una ley que apoya los principios de la reforma universitaria, pero eso dura muy poco, y se deja de lado todo lo que es la reforma. El APRA (Aliança Popular Revolucionária Americana) y la izquierda en general - Mariátegui es uno de los abanderados de la reforma universitaria - este movimiento recién logra cristalizarse en 1945 cuando se da una ley universitaria, en donde se recogen los principios básicos del movimiento reformista. Pero, en el año 48 hay un golpe militar, y dejan de lado todo, se da un retroceso y se vuelve a la antigua universidad oligárquica. Y hasta el año 1961 cuando el gobierno de Manuel Prado, que había tenido el apoyo del APRA, lleva adelante también una nueva ley universitaria, y esta ley universitaria recoge nuevamente los principios de la reforma. Yo estudié por ejemplo, con la ley del 61, porque se establecen las facultades, el cogobierno, la extensión universitaria, se democratiza la universidad. En realidad lo que el movimiento de Córdova planteaba, una de sus grandes reivindicaciones, era la democratización, en dos sentidos: uno, de aquellos que estaban excluidos, no solamente de un pequeño grupo de la oligarquía, como existía anteriormente; y dos, la democratización interna de la universidad, y para eso la participación de los estudiantes en el cogobierno.

La otra reivindicación, además de esta democrática, es la que plantea la búsqueda de una vinculación de la universidad con la sociedad, para ello se necesita modernizar la universidad, que una universidad moderna, universidad científica; tan es así que un grupo político que asume, de izquierda, los principios de la reforma universitaria hasta el día de hoy plantea la universidad científica y popular.

- Adelia Miglievich-Ribeiro: Una universidad que es de alto nivel científico $\mathrm{y}$, al mismo tiempo, popular es considerado por algunos una contradicción. Mi pregunta es cómo hacer converger la inclusión popular, el acceso a la universidad para el pueblo, y las avanzadas exigencias tecnológicas, innovación tecnológica, pensamiento de punta? Darcy pensó posible esta convergencia, ¿no? 
César Germaná: Bueno, Darcy Ribeiro lo plantea allí, decía que hay que permitir el acceso a un sector amplio de la población, hasta llegar a que todos los que están en edad para ingresar a la universidad, lo hagan. ¿Qué ocurre con aquellos que están mal formados? ¿La universidad debe tener cursos especiales para apoyarlos? O sea, en Argentina el ingreso es libre a la universidad, y la selección se hace a lo largo del primer año, y algo así creo que se encuentra la universidad necesaria de Darcy Ribeiro, yo no creo que sea contradictorio. Lo que ocurre, me parece a mí, es que el movimiento de la reforma universitaria se comienza a agotar porque se habían conseguido las dos reivindicaciones principales, la democratización que dio un incremento masivo de la matrícula universitaria. Cuando yo entré a la universidad en el año de 1961, había 40.000 estudiantes universitarios en el Perú y 8 universidades. Hoy hay 890.000 estudiantes universitarios y cerca de 144 universidades, o sea, ha habido una explosión de la matrícula, ha democratizado en el sentido de que lo que quería el movimiento de Córdova, y la otra reivindicación del gobierno de la universidad, también, prácticamente desde el año 84 hasta hoy, las universidades se han manejado a partir de la participación de los estudiantes como el cogobierno.

La masificación de la universidad y por otro lado la democratización del gobierno, no ha significado elevar la calidad académica, creo que a ese reto se enfrentó Darcy Ribeiro cuando plantea la reforma de la universidad. Ese es el reto que se planteaba, y creo que en el informe que hace al consejo superior de la universidad peruana, se van a plantear un modelo que permita elevar la calidad académica de la universidad. Allí es donde encuentro uno de los límites mayores de la propuesta de Darcy Ribeiro, porque lo que él plantea es incorporar la ciencia moderna a la universidad, es decir, que la universidad no esté al margen del desarrollo de las ciencias modernas. Y no cuestiona esa ciencia, esa manejar eurocéntrica de desarrollo de la ciencia, de otra manera uno no se explicaría la tragedia contemporánea, todo este complejo científico tecnológico está destruyendo a la humanidad, si se mantiene, la especie humana va a desaparecer, entonces tienes que haber otro tipo de conocimiento, otro tipo de ciencia. Eso no lo veía, creo yo, Darcy Ribeiro.

- Adelia Miglievich-Ribeiro: ¿Darcy Ribeiro no llegó a pensar en esto ni en su condición de antropólogo? Él llegó a ver la inclusión de pueblos indígenas, de lenguas indígenas en la universidad en su modelo de la aceleración evolutiva. Era casi una obsesión para Darcy Ribeiro que nosotros los latinoamericanos no podríamos quedarnos atrás con respecto 
de los estadounidenses de los europeos, la modernización refleja es nuestro peor enemigo...

- César Germaná: Me parece avanzar en lo creativo, no hay que basarse en esta modernización refleja, y por lo tanto la autonomía, pero esa autonomía no significaba romper con la ciencia europea o estadounidense, sino incorporarla. Yo creo que allí, inclusive en el libro de la Universidad necesaria, o de la Universidad nueva, porque allí hay una serie de ensayos, entre otros dos capítulos de un informe a este Conup, y en algún lugar de ese libro dice que uno de los obstáculos a un proceso creativo de evolución es el pensamiento indígena, dice que es importante para la identidad nacional, pero la universidad no lo puede incluir como base de su reforma, tiene que ser el desarrollo científico.

Y allí, hay dos cosas que yo creo que sería importantes: una que el modelo de ciencia ha sido cuestionado dentro del mundo europeo y estadounidense, la crisis de ese modelo newtoniano de ciencia, entonces aparece la nueva ciencia, la ciencia de la complejidad del caos del indeterminismo; entonces allí hay otro modelo de ciencia que se acerca más a los saberes de los pueblos originarios entonces que es un lugar de diálogo, un espacio de diálogo, posible, no como la antigua ciencia. Creo que eso no lo desarrolló Darcy Ribeiro, quería una universidad moderna pero autónoma. Por eso es congruente con el proyecto del régimen militar, que era el desarrollo de un capitalismo autónomo, y un desarrollo auto sostenido. En 1971 dieron la ley de industrias, y el primer artículo dice que el propósito de esa ley es el desarrollo de la industria para un desarrollo autónomo y auto sostenido, y creo que ese es el proyecto de Darcy Ribeiro.

- Adelia Miglievich-Ribeiro: La generación de Darcy Ribeiro, toda la generación de izquierda de este tiempo piensa en la modernización como la meta...

- César Germaná: Una cosa que tú dices en un artículo que me enviaste, hace algún tiempo ${ }^{1}$, es la influencia de Mannheim, se nota claramente que las ciencias humanas, como él llama, deben no solamente lograr la identidad nacional y trabajar por la identidad nacional sino por la planificación del desarrollo. Que es la idea de Mannheim, creo que es importante. No había reparado en esa influencia...

${ }^{1}$ Cesar Germaná refere-se à minha recepção do conceito de intelligentsia de Mannheim para pensarmos o intelectual público, sobretudo, dos anos 1950 no Brasil (Miglievich-Ribeiro, 2011). 
- Adelia Miglievich-Ribeiro: En Brasil es claro...

- César Germaná: En el Perú no. Mannheim nunca ha sido conocido, ni discutido, y eso tiene que ver con un hecho social; aquí en Perú entre 1939-1970 no hay una burguesía, que asuma este proyecto de un desarrollo nacional, como hay en Brasil.

- Adelia Miglievich-Ribeiro: ¿Pero cuando el gobierno revolucionario de Velasco comienza, no habría este proyecto de nación? ¿El comienzo de la idea de proyecto de sociedad?

- César Germaná: Como decía el sociólogo Julio Cotler, Velasco era un gobierno para la burguesía sin la burguesía. No para el pueblo, querían crear una burguesía porque no había una burguesía. Como lo que ocurre a partir de Kubitschek en Brasil, que es la internacionalización del mercado interno. Aquí también en los años 1950, la idea de un mercado interno autónomo, de un capitalismo nacional desaparece. Y es Velasco el que busca el desarrollo de un nuevo orden, autónomo, nuevo orden más auto sostenido, y por eso es que la Educación se presenta como un proyecto importante, y la universidad en primer lugar. Por eso es que apoya Darcy Ribeiro la propuesta de una reforma universitaria. Sin embargo, fue curioso pero en realidad contrariamente a las iniciativas, porque era el grupo intelectual que apoyaba a Velasco era de primer nivel. Salazar Bondi, Peñalosa eran filósofos que apoyaban la reforma a la educación, y quizá nunca antes o después se ha elaborado un proyecto de reforma a la educación tan radical como el de 1972.

- Adelia Miglievich-Ribeiro: Fue un proyecto muy radical e innovador, en mi opinión, a menos que haya sido solamente un discurso. En este libro "La universidad peruana", ellos hablan de "expansión universitaria", de "inclusión social", de que "todas las personas que quisieran estudiar podrían hacer cursos o disciplinas por separado, recibir certificados". Ellos hablam acerca de la articulación entre el gobierno, la universidad y el sistema productivo industrial. Una universidad para los trabajadores y sectores populares. ¿Es todo esto sólo un discurso?

- César Germaná: No es sólo un discurso, es también una serie de medidas que se adoptan como la reforma agraria, por ejemplo, la nacionalización de las principales empresas. En el año de 1975 cuando es desplazado Velasco por un golpe militar, el $66 \%$ de todo lo que se producía en bienes y servicios, lo producía el Estado, o sea, era el proyecto aprista de los años 30 de un capitalismo de estado. El complemento de ese proyecto era, 
en el caso del proyecto aprista de los años 1960, lo que ellos llamaban la democracia funcional, no la democracia liberal, sino funcional, y el velasquismo retoma eso como la democracia social de participación plena que implicaba considerar al individuo, o al ciudadano, no como cantidad, eso había dicho Haya de la Torre, sino como calidad el lugar que tiene en la división social del trabajo. Incorporar en el estado a las organizaciones sociales de base, era un proyecto en ese sentido radical, y no era solamente un discurso, se crearon la comunidad laboral, las ligas agrarias, y por lo tanto la educación tenía que acompañar eso que ellos llamaban "la revolución". Entonces era congruente una reforma de la educación en función de las necesidades de desarrollo permanente y auto sostenido y por eso, se crea no solamente la educación universitaria sino crea otro nivel previo, que es la educación superior técnica, la idea era después de la educación básica, un nivel intermedio para formar técnicos, que pudieran constituirse en la base de ese proceso de industrialización. Ya era coherente la reforma de la educación con la reforma del conjunto de la sociedad, y creo que eso es lo que valora Darcy Ribeiro.

- Adelia Miglievich-Ribeiro: ¿Cuáles son los intelectuales peruanos, usted ya dijo algunos nombres, que estaban dedicados a pensar la reforma universitaria?

César Germaná: En el ministerio de educación se formó la comisión de educación que publicó un pequeño libro, le llamaban el libro azul, de balance de lo que era la educación en el Perú. Esa comisión la dirigió un filósofo Augusto Salazar Bondy que era de alguna manera así como Enrique Dussel y desarrollo la idea de la filosofía de la liberación. Y otro educador y filósofo fue Walter Peñalosa. Creo que ellos eran las cabezas de ese grupo que estuvo en la reforma de la educación. Había otro sector de intelectuales que apoyaban el régimen militar, y por lo tanto, también todas estas medidas vinculadas a la educación que se agruparon en el Sinamos.

- Adelia Miglievich-Ribeiro: ¿Qué fue en verdad el Sinamos?

- César Germaná: Allí era un grupo de intelectuales que asesoraban al gobierno militar, y lo dirigió Carlos Delgado que era antropólogo, que había sido secretario personal de Haya de la Torre, y allí se trabajaron intelectuales como Carlos Franco, sociólogo y filósofo. Ese proyecto estaba vinculado a la autogestión yugoslava, su modelo era un modelo parecido al de Yugoslavia, por eso es que las cooperativas son las que van a reemplazar los latifundios en las cosas. Son formas de organización 
colectivas y en donde ya no hay esta propuesta de la reforma agraria como la división de la propiedad de la tierra, que después se va a lograr.

- Adelia Miglievich-Ribeiro: En Brasil, ni Getulio Vargas ni Juscelino Kubitschek se enfrentaron a los latifundistas pero sí Velasco. Me parece más cercano a la ideología estatal, socialista. ¿En ese sentido, los principales críticos del gobierno de Velasco eran contra la dictadura militar o a favor del capitalismo?

- César Germaná: Bueno el velasquismo tuvo dos sectores críticos. Uno la derecha que había perdido sus propiedades, los grandes latifundistas con la reforma agraria y después con la estatización de las empresas extranjeras. Y sobre todo cuando se estatizó la prensa, entonces perdieron no solamente las bases materiales, de su poder, sino las bases ideológicas. Por eso es que hasta ahora los sectores conservadores no le perdonan a Velasco el haber llevado adelante estas reformas, por eso lo siguen acusando de comunista. Dicen el régimen de Velasco fue comunista, y cuando quieren criticar a el Primer Ministro Ollanta Humala dicen que es un velasquista, un neovelasquista. Pero el otro sector crítico era de izquierda. El partido comunista más bien apoyó a Velasco, por ejemplo Velasco legalizó la confederación nacional de trabajadores del Perú, que estaba controlada por el partido comunista. En cambio, había un sector de intelectuales más autónomos, de los que te hablé que se reunían alrededor de la revista Sociedad y Política, que dirigía Aníbal Quijano. Quijano sostenía la tesis de que el régimen de Velasco era un régimen neo-imperialista, el neo-imperialismo que se asentaba aquí. Y Julio Cotler sostenía que políticamente era un corporativismo, porque el corporativismo es diferente al fascismo en la medida en que no destruye las organizaciones de los trabajadores. Claro que al final del régimen de Velasco, en 1974, ya surgió un sector dentro del velasquismo, con características fascistas.

- Adelia Miglievich-Ribeiro: Predichas por Quijano, ¿no?

- César Germaná: El movimiento laboral revolucionario que lo organizó el ministro de pesquería, Javier Tantalean, que destruía los sindicatos. Y por eso la izquierda del velasquismo se unió a la derecha, y dieron el golpe militar de 1975 que depuso a Velasco.

- Adelia Miglievich-Ribeiro: La derecha y la izquierda, los dos juntos derrocaron Velasco... 
- César Germaná: Dentro del régimen, eran militares de derecha y militares de izquierda. Morales Bermúdez, que era la derecha, Fernández Maldonado, que era la izquierda. Cuando publicaron en un periódico al día siguiente del golpe, en la primera página estaba Morales Bermúdez y en la última estaba Fernández Maldonado. La expresión de ese régimen.

- Adelia Miglievich-Ribeiro: Debía ser difícil para Darcy Ribeiro, un brasileño, exiliado, estar en una posición tan importante, en un momento en que la sociedad peruana estaba con tantos conflictos internos...

- César Germaná: Pero no creo, más bien me parece que frente a los militares, Castelo Branco, los militares argentinos, Pinochet, los militares peruanos eran exactamente lo opuesto, estaban haciendo una profunda transformación. En favor del pueblo, la Reforma agraria por ejemplo, ha sido una democratización y ya no se ha retrocedido, hay un neolatifundismo ahora pero ya no se ha retrocedido. Pero con Velasco se acabó la oligarquía, y los grandes terratenientes, los que Mariátegui denominaba gamonales, el gamonalismo.

- Adelia Miglievich-Ribeiro: ¿Los latifundios volvieron después de la caída de Velasco?

- César Germaná: No. En el gobierno de Alan García, el primer gobierno entre el 1985-90, lo que se hizo fue la división, la parcelación de las formas colectivas de propiedad de la tierra, o sea, se les entregó a los campesinos parcelas, se dividieron las cooperativas.

- Adelia Miglievich-Ribeiro: Perú está más avanzado que Brasil. Brasil mantiene sus latifundios.

- César Germaná: Lo que ocurre es que en América Latina ha habido dos grandes tendencias, una esta tendencia populista, como la de Vargas y la de Perón en Argentina, que era una alianza entre la burguesía nacional, las clases medias, los obreros asalariados, y de esa alianza los únicos excluidos eran los campesinos, por eso Vargas mismo era un gran terrateniente ¿no?

- Adelia Miglievich-Ribeiro: Si, Vargas puede ser adorado y odiado en diferentes momentos de la historia...

- César Germaná: Esa es una tendencia, la del populismo, como la de Vargas. La otra es el nacionalismo radical, que se concreta en la revolución boliviana del año 52, que lleva adelante la reforma agraria; o el gobierno 
de Jacobo Arias en Guatemala, que es el 54, que lleva también adelante la reforma agraria, y el Apra también aquí planteaba la reforma agraria, y por eso el velasquismo es la culminación de ese proceso, en donde se lleva adelante la reforma agraria, es tan radical o más radical inclusive que la de Chile de Allende, o casi como la de Cuba. En ese sentido, el velasquismo transformó profundamente la sociedad peruana. Entonces yo creo que Darcy Ribeiro se sentía cómodo dentro de un régimen militar, o sea, en la medida en que estaba logrando esas transformaciones, y por eso la necesidad de que la universidad se adecuara a esas transformaciones.

- Adelia Miglievich-Ribeiro: Cuando Velasco cae. ¿Cómo queda la universidad peruana, las propuestas de cambios de reestructuraciones?

César Germaná: Lo que ocurre en ese periodo con la universidad es muy extraño, en 1969 el régimen de Velasco da una ley por la que reorganiza la universidade. Se desaparecen las facultades, se establecen departamentos, donde están reunidos los profesores, y los llamados programas, que reúnen a los alumnos, que son las carreras profesionales. Y en la cúspide del gobierno de la universidad hay un concejo ejecutivo.

- Adelia Miglievich-Ribeiro: Después del fin del gobierno de Velasco, ¿Esto cambia?

- César Germaná: No cambia. Lo que ocurre es que eso se da en el 69, hay una oposición muy grande porque es un gobierno con una estructura burocrática autoritaria y desaparece la participación de los estudiantes. Todo lo que habían sido las conquistas de Córdova desaparecen. En 1972, se da una ley general de educación y esta ley tiene un capítulo sobre la educación superior y deja en manos de los propios universitarios que decidan cómo debe organizarse la universidad. Nunca antes, ni después, ha habido esta posibilidad de que los propios estudiantes y profesores decidan cómo organizar, entonces se convocó la ley del 72 que establecía la convocatoria a una asamblea estatutaria.

- Adelia Miglievich-Ribeiro: ¿Darcy Ribeiro formuló, junto con otros, esta ley del 1972?

- César Germaná: Si, él estuvo seguramente con la ley del 1972. Y entonces hay elecciones porque los representantes a la asamblea universitaria porque para estableces la nueva organización de la universidad se eligen por universidades. Yo participé por ejemplo en esa elección, pero perdí. La asamblea se reúne, $2 / 3$ profesores y $1 / 3$ estudiantes. Pero allí la influencia 
es de un grupo maoísta que es Patria Roja, ellos son los que tienen la mayoría de los representantes estudiantes, y parte de los profesores. Y elaboran un estatuto extremadamente radical así el gobierno no promulga el estatuto. Entonces la universidad desde el año 1972 hasta el año 1984, se va a gobernar por un estatuto de la ley de 1969, esa ley que había sido derogada. Es decir, en el año 1972 se deroga la ley de 1969, pero como no fue promulgado el nuevo estatuto, se mantiene el antiguo estatuto de la ley derogada. La universidad se va a mantener desde el 1972 hasta 1984, y hay una incertidumbre no hay un proyecto claro de las universidades.

- Adelia Miglievich-Ribeiro: Carreras docentes, currículos...

- César Germaná: Yo comencé a enseñar con esa ley en 1970. Todo estaba legislado por el estatuto de 1969, entonces no había participación de los estudiantes, surge un grupo mayoritario sobre todo en universidades públicas, en San Marcos, que dice que este es un gobierno fascista, y no quieren participar; porque el estatuto plantea la participación de los estudiantes del tercio superior con una serie de limitaciones que los estudiantes no aceptan.

- Adelia Miglievich-Ribeiro: Yo podría decir que el pensamiento crítico acerca de la universidad latinoamericana fue efervescente? Diversas personas participaron de este debate, aunque en la práctica este pensamiento crítico no fue implantado.

- César Germaná: No, porque cuando se da la nueva ley universitaria en 1984, que rige hasta ahora, ya después de la caída del régimen militar. Con el gobierno de Francisco Belaúnde Terry el se da una nueva ley universitaria en 1983. Y ahí lo que se recoge son las reivindicaciones de la reforma universitaria, cogobierno, la cátedra paralela, la proyección social. Se vuelven a las facultades...

- Adelia Miglievich-Ribeiro: ¿Usted piensa que es importante para nosotros continuar hablando sobre la universidad necesaria hoy, a pesar de todos los problemas que fue narrado?

- César Germaná: Sí. En este momento hay una gran discusión aquí en el Perú porque se ha planteado en el congreso una nueva ley universitaria, la comisión de educación de este congreso ya aprobó el dictamen, y falta discutirlo en el pleno, entonces esto ha generado un debate muy grande. Pero es un debate superficial porque afecta algunos aspectos que no son 
los fundamentales, para plantearse el problema de la universidad hoy. Qué se discute, sobre todo, ya el proyecto aprobado por la comisión de educación señala la formación de una superintendencia que controle a las universidades. En el Perú ahora hay tres tipos de universidades: la universidad pública que es financiada por el estado, la universidad privada sin fines de lucro, y la universidad privada con fines de lucro, es decir, las universidades empresa. Esos son los tres tipos, y lo que ha crecido más es esta universidad empresa, la mayor parte de las universidades ahora son estas empresas, que son de muy baja calidad, porque lo que les interesa es ganar dinero, y como hay una demanda muy grande por los estudios universitarios, entonces estas empresas lucran con la educación. Lo que critican estas universidades sobre todo es lo que ellos señalan la falta de autonomía de la universidad, que se pone en cuestión la autonomía de la universidad.

- Adelia Miglievich-Ribeiro: Sí, si el objetivo es el lucro...

- César Germaná: Claro, porque lo que buscan es pagarle lo menos posible al profesor, y cobrarle la mayor pensión a los alumnos. Ahí está el negocio de esta universidad. En la pública la objeción es porque este nuevo proyecto plantea la elección por votación universal de las autoridades. Actualmente se eligen al rector y a los decanos a través de un concejo de facultad y una asamblea universitaria. En esos concejos y asambleas hay $2 / 3$ de profesores y $1 / 3$ de estudiantes, pero el sistema se ha corrompido.

- Adelia Miglievich-Ribeiro: ¿Quiénes son los corruptores?

- César Germaná: Los mismos grupos de la universidad pero de los que tienen dinero y entonces compran el voto de los estudiantes, entonces se ha corrompido el cogobierno que era la demanda principal de la reforma universitaria; ha llevado a la formación de pequeñas mafias, que son las que gobiernan las universidades públicas, y ellos son los que se oponen a que se lleve adelante una elección por votación universal, pero no se pone en cuestión la relación entre universidad y el poder. Eso decía Darcy Ribeiro, él decía bien claramente que hay una articulación entre la universidad y la estructura de poder, porqué la universidad es conservadora, porque está al servicio de una forma de poder de los grupos oligárquicos o burgueses. Para que haya un cambio en la universidad tiene que haber un cambio en la sociedad, y por eso él veía ese cambio en el régimen militar y la universidad debería adecuarse a esos cambios, o sea, no puede haber una universidad que sea una isla. Este problema es el que no se ha examinado. 
- Adelia Miglievich-Ribeiro: Sin cambiar la sociedad, la universidad va a mantenerse conservadora...

- César Germaná: Y es lo que planteaba Darcy Ribeiro, estos cambios en la sociedad necesariamente tenían que llevar a que la universidad se pusiera al servicio de estas transformaciones, al servicio del pueblo, como él dice. Ahora, es cierto también, que aunque no cambia la sociedad, la universidad aún puede cambiar, puede tener alguna influencia sobre la sociedad pero eso implica cuestionar esta forma de colonialismo en las estructuras de saber que se imponen; por eso la idea descolonialidad de la universidad.

- Adelia Miglievich-Ribeiro: ¿Cómo está este debate de la descolonialidad del saber hoy?

- César Germaná: Esta en los inicios porque no hay una conciencia de la necesidad de cuestionar estas formas eurocéntricas de saber, que es lo que se encuentra en Mariátegui por ejemplo. Mariátegui tenía una visión que ahora podríamos llamarla decolonial, a mí más me gusta descolonialidad que decolonial, que es una influencia del inglés. Bueno a Quijano también una vez le pregunté qué prefería decolonial o descolonialidad, y dijo que descolonialidad.

- Adelia Miglievich-Ribeiro: ¿Cuál es su opinión acerca del pensamiento de Walter Mignolo?

- César Germaná: Me parece a mí que ha propuesto esta diferencia colonial que creo que da cuenta de la existencia de este pensamiento fronterizo, o epistemología fronteriza, creo que constituye uno de los puntos de referencia fundamental. El año pasado hubo una reunión en la Universidad Federal de Rio de Janeiro (UFRJ). Fue muy interesante. Estaban presentes Alberto Acosta, Catherine Walsh, que está en la Universidad de Bolívar de Ecuador de Quito, Edgardo Lander, que está en la Universidad Central de Venezuela.

- Adelia Miglievich-Ribeiro: ¿Esta perspectiva esta influencia tiende a crecer?

C César Germaná: Aquí en Perú la influencia es mínima.

- Adelia Miglievich-Ribeiro: ¿Cómo podría ser ampliado este debate en Perú? Específicamente acerca de la descolonización de la universidad. ¿Con la internacionalización del debate? 
- César Germaná: Yo creo que una de las formas sería la publicación de algunos materiales, por ejemplo, Lander ha escrito algo sobre eso y Santiago Castro Gómez también, que son algunos textos fundamentales que no se conocen, entonces la idea es difundir este material. Lo otro es plantearse un material de investigación de más grande aliento.

- Adelia Miglievich-Ribeiro: Incluyendo el conocimiento de los pueblos indígenas, los las personas de ascendencia africana y sus voces porque no puede ser la repetición de nuestros discursos y materiales convencionales...

- César Germaná: No, por ejemplo, la Universidad intercultural de las nacionalidades y pueblos indígenas "Amawtay Wasi" de Ecuador es una experiencia bien interesante porque esto muestra como hay en estos gobiernos llamados de izquierda en América Latina dos perspectivas: una de un neodesarrollismo estrativista, de Evo Morales; y por otro lado la vertiente más vinculada a los pueblos indígenas que plantea la ideología del "buen vivir", de suma causa. Son dos orientaciones y esto se muestra en la modernización de la universidad ecuatoriana. El gobierno ecuatoriano ha dado cantidad de dinero, está apoyando la universidad pero una universidad que quiere convertirse en eso que Darcy Ribeiro llama la modernidad refleja...

- Adelia Miglievich-Ribeiro: La economía neoestrativista...

- César Germaná: Para hacer de la universidad una universidad del primer mundo, y han evaluado a las universidades y han cerrado la universidad indígena.

- Adelia Miglievich-Ribeiro: Estás experiencias en Ecuador, y otras experiencias de universidades pluriétnicas podrían ser estudiadas? ¿No podría ser un programa de estudio, de investigación? ¿Ya existen tales estudios?

- César Germaná: Yo no conozco por lo menos. Pero habría que hacer un balance de estas nuevas universidades. Yo estoy ahora interesado en realizar un proyecto entre las nuevas relaciones sobre la universidad y el poder. Cómo se ha desenvuelto esa relación, cómo se ha dado, y cuál sería la posibilidad de que estas universidades se logren descolonizar. Ya debería haberlo comenzado. Sería antes de despedirme de la universidad porque me pienso jubilar. 
Y esto lo veía bien Darcy Ribeiro, que la universidad está vinculada al poder, los profesores los intelectuales, esas élites están al servicio del poder, y cómo el poder los coopta, y se los come. México es un ejemplo de eso, pueden tener un lenguaje radical...

- Adelia Miglievich-Ribeiro: ¿El pensamiento de Darcy Ribeiro es conocido en Perú?

- César Germaná: Creo que es muy marginal, ni siquiera el pensamiento antropológico que debería ser más influyente, por ejemplo "El proceso civilizatorio".

- Adelia Miglievich-Ribeiro: ¿Nosotros podemos hablar hoy de identidad latinoamericana? ¿De modo descolonial?

- César Germaná: Yo creo que sí tenemos una herencia común y tenemos un destino común, estuve leyendo hace poco un artículo en una página de internet que había recuerdos de Leopoldo Zea sobre Darcy Ribeiro, que fueron muy amigos, le llevaba 10 años y le afectó mucho la muerte de Darcy Ribeiro.

- Adelia Miglievich-Ribeiro: Una pregunta para mi curiosidad. ¿Existe en Perú la política de acción afirmativa para el ingreso en la universidad? ¿Cuotas raciales, por ejemplo?

- César Germaná: Desde el año 98 en San Marcos había una política de apoyo a los estudiantes que venían de los pueblos indígenas, el requisito era haber terminado la educación básica, y hablar la lengua de su pueblo, materna. Eso se mantuvo y fue un fracaso total. Por ejemplo hay un pintor que estudió historia y que murió. Bueno en general la universidad está hecha para los grupos urbanos y sectores medios, en cambio llega un joven de algún grupo de la selva y se pierde, yo he tenido alumnos. Todos han fracasado... Hay un caso, fui a Bagua que está en el departamento del amazonas, y allí me encontré a una enfermera que había ingresado a San Marcos por este sistema de apoyo a la población indígena, y así se había desenvuelto bien, y como sabía la lengua entonces era una mediadora entre occidente y su pueblo, esto fue bien interesante pero en general no han tenido mucho éxito.

Me imagino que es difícil ubicar un proceso tan complejo como ese del Perú, que se diferencia bastante del de Brasil, somos vecinos pero somos muy diferentes. 
- Adelia Miglievich-Ribeiro: Estoy muy agradecida por su entrevista. Para mí fue una clase, muchas gracias.

\section{Referência}

MIGLIEVICH-RIBEIRO, Adelia Maria. Darcy Ribeiro e o enigma Brasil: um exercício de descolonização epistemológica. Revista Sociedade e Estado, v. 26, n. 2 , p. 23-49, 2011.

Recebido em: 6 jun. 2015

Aprovado em: 30 set. 2015

Autora correspondente:

Adelia Miglievich-Ribeiro

Av. Fernando Ferrari, 514 - Goiabeiras

29075-910 Vitoria, ES, Brasil 